Chapter 16

\title{
EMG-Controlled Prosthetic Hand with Fuzzy Logic Classification Algorithm
}

\author{
Beyda Taşar and Arif Gülten \\ Additional information is available at the end of the chapter
}

http://dx.doi.org/10.5772/intechopen.68242

\begin{abstract}
In recent years, researchers have conducted many studies on the design and control of prosthesis devices that take the place of a missing limb. Functional ability of prosthesis hands that mimic biological hand functions increases depending on the number of independent finger movements possible. From this perspective, in this study, six different finger movements were given to a prosthesis hand via bioelectrical signals, and the functionality of the prosthesis hand was increased. Bioelectrical signals were recorded by surface electromyography for four muscles with the help of surface electrodes. The recorded bioelectrical signals were subjected to a series of preprocessing and feature extraction processes. In order to create meaningful patterns of motion and an effective cognitive interaction network between the human and the prosthetic hand, fuzzy logic classification algorithms were developed. A five-fingered and 15-jointed prosthetic hand was designed via SolidWorks, and a prosthetic prototype was produced by a 3D printer. In addition, prosthetic hand simulator was designed in Matlab/SimMechanics. Pattern control of both the simulator and the prototype hand in real time was achieved. Position control of motors connected to each joint of the prosthetic hand was provided by a PID controller. Thus, an effective cognitive communication network established between the user, and the real-time pattern control of the prosthesis was provided by bioelectrical signals.
\end{abstract}

Keywords: EMG, fuzzy logic classification, multifunctional prosthesis hand, pattern recognition

\section{Introduction}

People lose limbs due to accidents and medical conditions. Robotic devices, which imitate the shape and function of a missing limb, are manufactured for use by people who lose their limb in such situations. In recent years, researchers have studied to design and control multifunctional 
prosthetics hand [1-7]. The complexity of the movement, that is, the number of independent movements, increases in proportion to the number of joints. There are 206 bones in the adult skeletal system. The 90 bones of the skull and face are connected to each other by nonimmobilized joints, and the 33 bones of the spine are connected to each other by semi-movable joints. Movable joints are only present between the bones (except the metacarpals bones) of the arm (25) and leg (25). In light of this information, aside from the wrist joints, the human hand has 15 independent joints with three on each finger. Therefore, the biological hand movement involves the control of these joints independently. Thus, control of the hand is quite complex. Thus, of all the human parts, the hand is the most complicated in terms of kinetic analysis [8].

Two main factors enable the functional and visual prosthesis to be used like a biological hand:

- Prosthetic hand mechanical design and modeling $[9,10]$ and

- $\quad$ Perform the position and speed controls of each joint efficiently and precisely [11-19].

However, no matter how perfect the design and manufacture of the prosthetic hand may be, the utility depends on the cognitive interaction, i.e., the control algorithm, being designed properly, e.g., the type of movement and coordination between fingers. If information is not transferred to the prosthetic hand rapidly enough, then the prosthesis will not assume the desired position. Cognitive interaction is the most important factor for user to use effectively. There are many studies about cognitive interaction between human and robotic devices [20-25].

All voluntary muscle movements in humans occur as a result of bioelectrical signals transmitted from the brain through the muscle nerves. Bioelectrical electromyogram (EMG) signals transmitted to the muscles carry information about the type of movement, speed, and degree of muscle contraction or relaxation. The biological hand performs the basic tasks of holding and gripping, which involve various finger movements. The wrist movements essentially constitute the axis and assist in these gripping and holding movements. The main factor that increases the functionality of the prosthetic hand is the movement of the fingers. As the number of independent movements made by the prosthetic hand increases, it can mimic the biological hand more successfully. This study realizes the design of the bioelectrical signal control algorithm and the extension of the bioelectrical signal database with the purpose of increasing the finger motion function of bioelectrical signal-controlled prosthesis hands.

Figure 1 shows bioelectrical signals in the context of the activity of the muscle movements (e.g., flexion, relaxation force), as seen from the block flow diagram. EMG can be used to detect signals from the flexor pollicis longus, flexor carpi radialis, brachioradialis, extensor carpi radialis, extensor digiti minimi, and extensor carpi ulnaris. Bioelectrical signals were recorded with the help of four surface electrodes and subjected to a series of preprocessing and classification operations to understand the relationships between EMG signals and hand and finger movements. These signals were then applied to the prosthetic hand (space and simulator) as a reference motion signal. With the designed controllers, the position of the prosthetic hand finger joints can be controlled. Thus, a cognitive interface and communication network are established between the user and the prosthetic hand. Briefly summarized, the study creates a bioelectrical database of the activities of the hand muscles and the interaction network between the human and prosthetic hand using this database and interface to design a simulator and develop a control algorithm. 


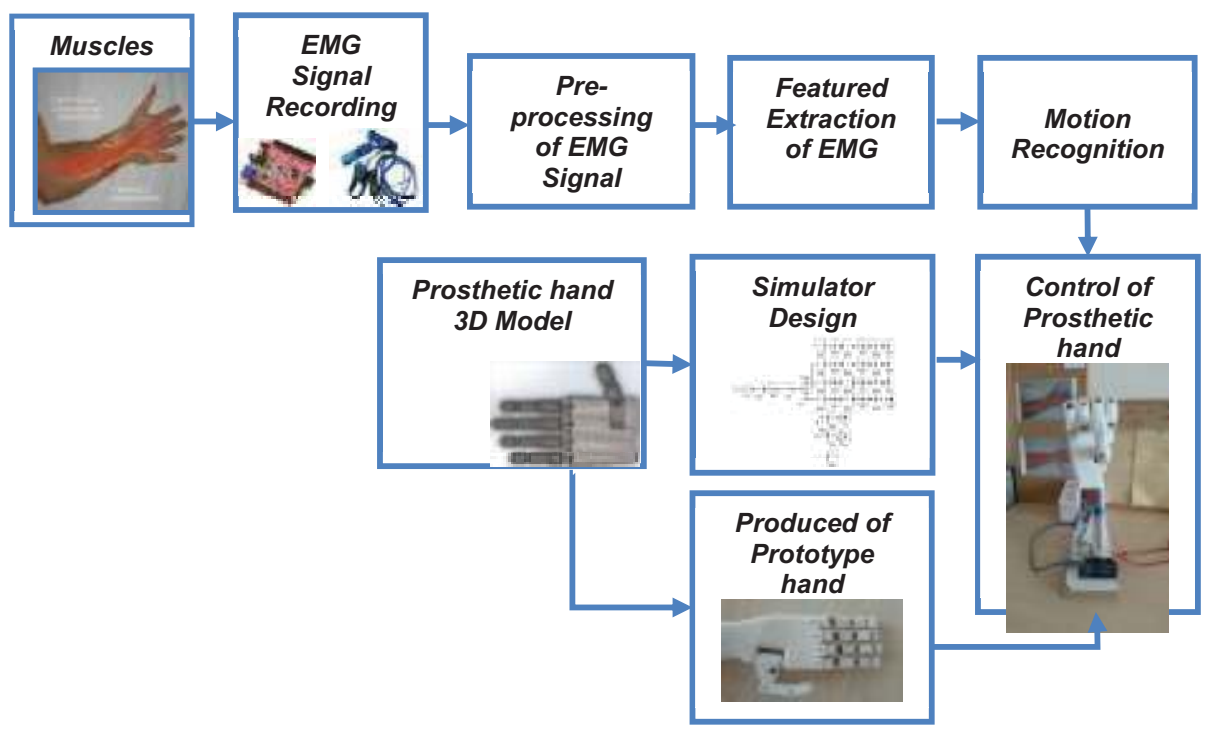

Figure 1. Control of multifunctional prosthetic hand simulator and prototype with EMG signals.

\section{Recording, preprocessing, and featured extractions of EMG signal}

\subsection{Recording of EMG signals}

EMG signals were recorded from the forearm muscles (the flexor pollicis longus, flexor carpi radialis, brachioradialis, extensor carpi radialis, extensor digiti minimi, and extensor carpi ulnaris) with the help of four surface electrodes. Electrode placements are shown in Figure 2. Electrode layout was chosen according to the protocol [26-28].

The signals, which support movements of the thumb, middle, ring, index, and pinkie fingers, were recorded separately for each of the respective muscles. Channels and finger relations are shown in Table 1.

\subsection{Preprocessing of EMG signals}

The recorded EMG signals also include various noise signals. It is necessary to separate the noise signals from the EMG signals, so that the characteristics of the signal can be accurately
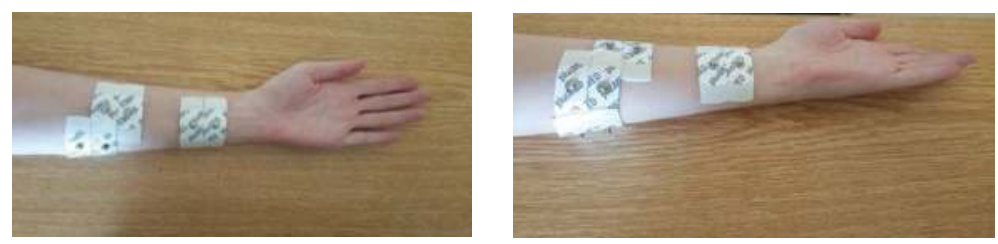

Figure 2. Placement of surface electrodes. 


\begin{tabular}{llll}
\hline Channel 1 & Channel 2 & Channel 3 & Channel 4 \\
\hline Pinkie finger muscle & Ring finger muscle & Middle finger muscle & İndex finger muscle \\
\hline
\end{tabular}

Table 1. Channel finger relations.

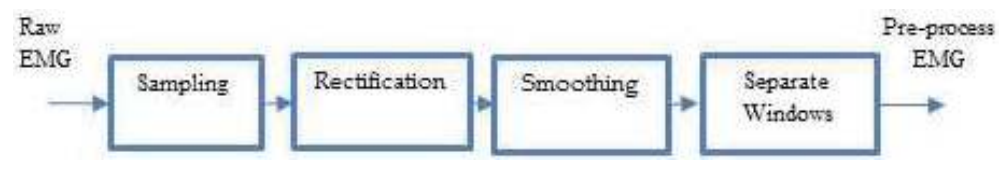

Figure 3. Preprocessing steps.

determined. For this reason, the raw EMG signal is first preprocessed. The block diagram of the preliminary preparation stage, including the separation, rectification, and sampling of the recorded EMG signals from noise, is shown in Figure 3.

\subsubsection{Numerical sampling}

EMG signals are analog voltage signals. Their amplitudes change constantly over the voltage range. Analog-to-digital conversion is the process by which the amplitude of the analog signal voltage is represented by a number sequence at specific time points [29-31]. The EMG voltage signals used in this study are converted into a number sequence by sampling with a period of $0.001 \mathrm{~s}$.

\subsubsection{Rectification process}

Rectification is the evaluation of only the positive parts of the signal. This is done either by halfwave or full-wave rectification of the signal. A full-wave rectification method was applied to preserve the energy of the signal [25, 29-34], and the expression for the method is given in Eq. (1).

$$
X_{\text {_training }}=|\mathrm{x}(\mathrm{t})|
$$

\subsubsection{Smoothing of signal}

A bandpass filter $(50-500 \mathrm{~Hz})$ was designed to soften the signal by eliminating high-frequency components.

\subsubsection{Separate the signal into windows}

Before the attributes of the obtained EMG signals are calculated, the frame is processed by the method adjacent to the signal. Experiments in the study of Englehart $[18,19]$ for framing and optimal framing values $(\mathrm{R}=256, \mathrm{r}=32 \mathrm{~ms})$ reached with calculations were used.

\subsection{Featured extractions of EMG signal}

The EMG signal is a non-stationary, time-varying signal that varies in amplitude by random negative and positive values [25, 31, 32]. Bioelectrical signals have certain characteristic values, i.e., information. Features in time domain have been widely used in medical and engineering practices and researches. Time domain features are used in signal classification due to its easy 
and quick implementation. Furthermore, they do not need any transformation, and the features are calculated based on raw EMG time series. Moreover, much interference that is acquired through the recording because of their calculations is based on the EMG signal amplitude. However, compared to frequency domain and time-frequency domain, time domain features have been widely used because of their performances of signal classification in low noise environments and their lower computational complexity [29]. In this study, five time domain features methods widely used in the literature have been utilized to obtain the features of the EMG signal.

\subsubsection{Signal energy}

Mathematically, the energy of the signal $m(t)$ is calculated as in Eq. (2), where $t_{j}$ and $t_{i}$ denote the lower and upper bounds of the part of the signal to be integrated, respectively. The above expression represents the area below the absolute value of the signal curve at time $T=t_{i}-t_{j}$ [30-35].

$$
E=\int_{t_{i}}^{t_{j}}|m(t)| d t
$$

\subsubsection{Maximum value of signal}

The maximum value of the signal represents the largest of the sampled signal values in each packet divided by windows [29].

\subsubsection{Signal average value}

Mathematically, the average of the signal $m(t)$ is calculated as Eq. (3) [30, 31], where $t_{i}$ and $t_{j}$ denote the upper and lower bounds of the part of the signal to be integrated, respectively. The above expression represents the overall average of the signal at time interval $T=t_{i}-t_{j}$.

$$
A V R=\frac{1}{t_{j}-t_{i}} \int_{t_{i}}^{t_{j}}|m(t)| d t
$$

\subsubsection{Effective value of the signal}

Effective value is a commonly used signal analysis method in the time domain, such as average rectification [29-32]. The effective value of the $m(t)$ signal is calculated as Eq. (4).

$$
R M S=\left(\frac{1}{T} \int_{0}^{t} m^{2}(t) d t\right)^{\frac{1}{2}}
$$

\subsubsection{Variance of signal}

The variance value of the signal represents the amount of deviation from the mean of the sampled signal values in each packet divided by windows [30]. $p(t)$ is the variance of the signal to represent the probability density function of $t$ :

$$
V A R=\left(\frac{1}{T} \int_{0}^{t}(x-O R T)^{2} p(t) d t\right)
$$




\section{Pattern recognition with fuzzy logic algorithm}

A classifier's function should be able to map different patterns, match them appropriately, and, in this case, select different hand grip postures. The extracted features were then fed into the fuzzy logic (FL) classifier for the developed control system. FL developed by Lofty Zadeh [35-41] provides a simple way to arrive at a definite conclusion based solely on imprecise input information. A summary of the feature extraction process from the forearm muscles is shown in Table 2 according to motion.

In total, there are 20 features of EMG signal for four channels. In order to make relations easier, a featured function, which occurs at RMS, AVR, MAX, VAR, and E values, is defined for each channel. Finally, the number of inputs is reduced by four. The featured function is calculated as follows in Eq. (6).

$$
F_{i}=E_{i}+A V R_{i}+M A X_{i}+V A R_{i}+R M S_{i}
$$

For the FL classification analysis, the triangular shape of the membership function (MF) for the inputs $\left(F_{i}\right)$ and output and the centroid method for defuzzification are used. The rules are created

\begin{tabular}{|c|c|c|c|c|c|c|c|}
\hline & Signal & $\begin{array}{l}\text { Hand } \\
\text { closure }\end{array}$ & $\begin{array}{l}\text { Hand } \\
\text { opening }\end{array}$ & $\begin{array}{l}\text { Index-thumb } \\
\text { touch }\end{array}$ & $\begin{array}{l}\text { Middle-thumb } \\
\text { touch }\end{array}$ & $\begin{array}{l}\text { Ring-thumb } \\
\text { touch }\end{array}$ & $\begin{array}{l}\text { Pinky-thumb } \\
\text { touch }\end{array}$ \\
\hline \multirow[t]{4}{*}{ Energy } & Channel 1 & 16,41091 & 9,949203 & 5,853087 & 5,405963 & 5,354211 & 12,84222 \\
\hline & Channel 2 & 12,48169 & 10,92331 & 7,334108 & 6,46115 & 13,25441 & 5,029002 \\
\hline & Channel 3 & 12,02946 & 9,254157 & 8,313991 & 12,82708 & 7,183281 & 4,252198 \\
\hline & Channel 4 & 14,59524 & 7,548085 & 11,22431 & 6,920272 & 9,376161 & 4,381767 \\
\hline \multirow[t]{4}{*}{ Maximum value } & Channel 1 & 2,378095 & 1,398911 & 0,822295 & 0,61429 & 0,725287 & 2,255524 \\
\hline & Channel 2 & 1,674114 & 1,183987 & 1,126519 & 0,961061 & 1,90971 & 0,609637 \\
\hline & Channel 3 & 1,606747 & 1,351835 & 1,163335 & 1,60762 & 1,147475 & 0,666139 \\
\hline & Channel 4 & 1,990469 & 0,844166 & 1,437937 & 0,906574 & 1,485923 & 0,532234 \\
\hline \multirow[t]{4}{*}{ Average value } & Variance & 0,656436 & 0,397968 & 0,234123 & 0,216239 & 0,214168 & 0,513689 \\
\hline & Channel 1 & 0,499268 & 0,436932 & 0,293364 & 0,258446 & 0,530176 & 0,20116 \\
\hline & Channel 2 & 0,481178 & 0,370166 & 0,33256 & 0,513083 & 0,287331 & 0,170088 \\
\hline & Channel 3 & 0,58381 & 0,301923 & 0,448973 & 0,276811 & 0,375046 & 0,175271 \\
\hline \multirow[t]{4}{*}{ RMS value } & Channel 4 & 0,474695 & 0,273057 & 0,163739 & 0,134428 & 0,148438 & 0,387735 \\
\hline & Channel 1 & 0,325763 & 0,25909 & 0,207215 & 0,173207 & 0,370618 & 0,124443 \\
\hline & Channel 2 & 0,316673 & 0,25826 & 0,223731 & 0,339657 & 0,213173 & 0,122159 \\
\hline & Channel 3 & 0,383453 & 0,188114 & 0,295885 & 0,180392 & 0,269928 & 0,10675 \\
\hline \multirow[t]{4}{*}{ Variance } & Channel 4 & 0,72476 & 0,223357 & 0,08254 & 0,045411 & 0,066981 & 0,508143 \\
\hline & Channel 1 & 0,293061 & 0,15076 & 0,133987 & 0,086676 & 0,422607 & 0,038505 \\
\hline & Channel 2 & 0,281122 & 0,204654 & 0,145503 & 0,326644 & 0,150682 & 0,047588 \\
\hline & Channel 3 & 0,410777 & 0,089351 & 0,246002 & 0,089669 & 0,232966 & 0,027352 \\
\hline
\end{tabular}

Table 2. Summary of the feature extraction process from the forearm muscles. 
based on information from the states of contraction. FLC rules are shown in Table 3. Recorded SEMG signals have been used to initial testing. Then real time data implemented to Prosthetic hand model.

Fi Featured functions were inputs to the FL. The limits of F were set to [0, 20]. The three linguistic variables used were Small (S), Medium (M), and Big (B). The outputs of FL were Hand closure, Hand opening, Index-thumb contact, Middle-thumb contact, Ring-thumb contact, and Pinkythumb contact. Figure 4 shows the flow diagram of FL classification process from four SEMG signals for six hand patterns [35].

\begin{tabular}{llllll}
\hline Rules & F1 & F2 & F3 & F4 & Result \\
\hline 1 & BIG & BIG & BIG & BIG & Hand closure \\
2 & MEDIUM & MEDIUM & MEDIUM & MEDIUM & Hand opening \\
3 & MEDIUM & MEDIUM & MEDIUM & BIG & Index-thumb touch \\
4 & MEDIUM & MEDIUM & BIG & MEDIUM & Middle-thumb touch \\
5 & MEDIUM & BIG & MEDIUM & MEDIUM & Ring-thumb touch \\
6 & BIG & MEDIUM & MEDIUM & MEDIUM & Pinky-thumb touch \\
7 & SMALL & SMALL & SMALL & SMALL & Relax-no motion \\
\hline
\end{tabular}

Table 3. FL rules.

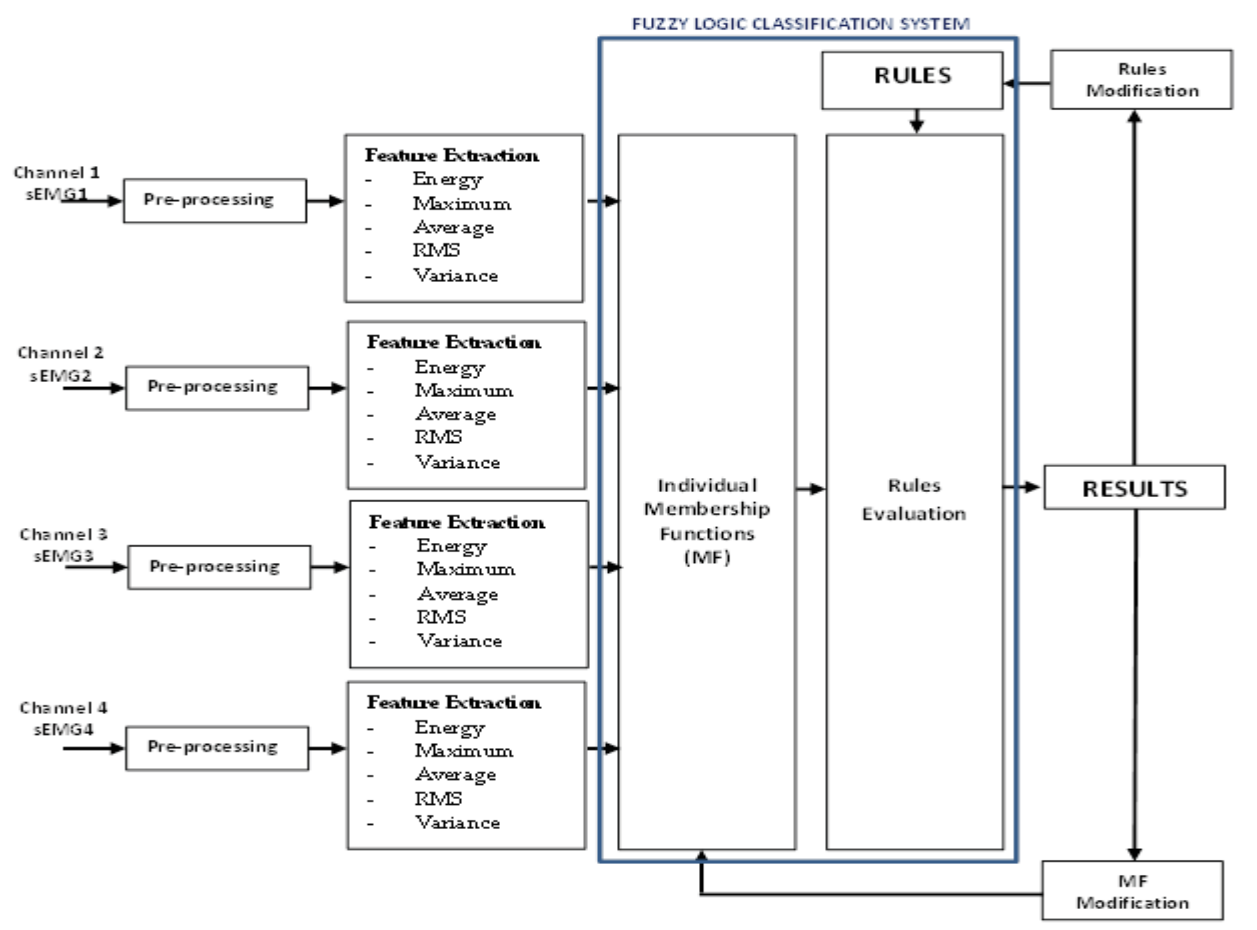

Figure 4. The flow diagram of the control system with FL classification components. 
Performance of FL tested 200 hand motions. Classification performance value for the six motions is shown in Table 4 .

In the medical decision-making process, ROC analysis method is used to determine the discrimination of the test or classification algorithm. In this study, performance of FLC algorithm for six motion class are demonstrated in Table 5 via ROC analysis.

Performance values calculated as Eqs. (7)-(10) for each hand motion

$$
\begin{gathered}
\text { Accuracy }(A C C)=\Sigma \text { True positive }+\Sigma \text { True negative } / \Sigma \text { Total population } \\
\text { Positive predictive value }(P P V) \text {, Precision }=\Sigma \text { True positive } / \Sigma \text { Test out comepositive } \\
\text { True positive rate }(T P R), \text { Sensitivity }=\Sigma \text { True positive } / \Sigma \text { Condition positive }
\end{gathered}
$$

False positive rate $(F P R)=\Sigma$ False positive $/ \Sigma$ Condition negative

\begin{tabular}{|c|c|c|c|c|c|c|}
\hline \multirow{2}{*}{$\begin{array}{l}\text { ROC analysis } \\
\text { Classification } \\
\text { algorithm result }\end{array}$} & \multicolumn{6}{|l|}{ Motions } \\
\hline & $\begin{array}{l}\text { Hand } \\
\text { closure }\end{array}$ & $\begin{array}{l}\text { Hand } \\
\text { opening }\end{array}$ & $\begin{array}{l}\text { Index-thumb } \\
\text { touch }\end{array}$ & $\begin{array}{l}\text { Middle-thumb } \\
\text { touch }\end{array}$ & $\begin{array}{l}\text { Ring-thumb } \\
\text { touch }\end{array}$ & $\begin{array}{l}\text { Pinky-thumb } \\
\text { touch }\end{array}$ \\
\hline Hand closure & 84 & 0 & 0 & 0 & 0 & 0 \\
\hline Hand opening & 0 & 84 & 0 & 0 & 0 & 0 \\
\hline Index-thumb touch & 0 & 0 & 76 & 6 & 6 & 4 \\
\hline Middle-thumb touch & 0 & 0 & 1 & 66 & 2 & 0 \\
\hline Ring-thumb touch & 0 & 0 & 4 & 10 & 72 & 3 \\
\hline Pinky-thumb touch & 0 & 0 & 2 & 0 & 1 & 76 \\
\hline No motion & 0 & 0 & 1 & 2 & 1 & 1 \\
\hline
\end{tabular}

\begin{tabular}{llllll}
\hline Hand pattern & $\begin{array}{l}\text { Pattern } \\
\text { number }\end{array}$ & $\begin{array}{l}\text { Tested total number } \\
\text { of motion (A+ B) }\end{array}$ & $\begin{array}{l}\text { Number of true } \\
\text { classified motion (A) }\end{array}$ & $\begin{array}{l}\text { Number of wrong } \\
\text { classified motion (B) }\end{array}$ & $\begin{array}{l}\text { Average } \\
\text { percentage of } \\
\text { success (\%) }\end{array}$ \\
\hline Hand closure & MOTION 1 & 84 & 84 & 0 & 100 \\
Hand opening & MOTION 2 & 84 & 84 & 0 & 100 \\
Index-thumb touch & MOTION 3 & 84 & 76 & 8 & 90.476 \\
Middle-thumb touch & MOTION 4 & 84 & 66 & 18 & 78.57 \\
Ring-thumb touch & MOTION 5 & 84 & 72 & 12 & 85.714 \\
Pinky-thumb touch & MOTION 6 & 84 & 76 & 8 & 90.476 \\
\hline
\end{tabular}

Table 4. Classification achievement percentages.

Table 5. ROC analysis. 


\begin{tabular}{|c|c|c|c|c|c|c|c|c|}
\hline \multicolumn{3}{|c|}{ Hand closure } & \multicolumn{3}{|c|}{ Hand opening } & \multicolumn{3}{|c|}{ Index -thumb touch } \\
\hline $\mathrm{TP}=84$ & $\mathrm{FN}=0$ & 84 & $\mathrm{TP}=84$ & $\mathrm{FN}=0$ & 84 & $\mathrm{TP}=76$ & $\mathrm{FN}=16$ & 92 \\
\hline $\mathrm{FP}=0$ & $\mathrm{TN}=420$ & 420 & $\mathrm{FP}=0$ & $\mathrm{TN}=420$ & 420 & $F P=8$ & $\mathrm{TN}=404$ & 412 \\
\hline 84 & 420 & 504 & 84 & 420 & 504 & 84 & 420 & 504 \\
\hline \multicolumn{3}{|c|}{$\mathrm{TPR}=1.00$} & \multicolumn{3}{|c|}{$\mathrm{TPR}=1.00$} & \multicolumn{3}{|c|}{$\mathrm{TPR}=0.826$} \\
\hline \multicolumn{3}{|c|}{$F P R=0.00$} & \multicolumn{3}{|c|}{$F P R=0.00$} & \multicolumn{3}{|c|}{$F P R=0.0194$} \\
\hline \multicolumn{3}{|c|}{$P P V=1.00$} & \multicolumn{3}{|c|}{$P P V=1.00$} & \multicolumn{3}{|c|}{$P P V=0.904$} \\
\hline \multicolumn{3}{|c|}{$A C C=1.00$} & \multicolumn{3}{|c|}{$A C C=1.00$} & \multicolumn{3}{|c|}{$A C C=0.952$} \\
\hline \multicolumn{3}{|c|}{ Middle -thumb touch } & \multicolumn{3}{|c|}{ Ring -thumb touch } & \multicolumn{3}{|c|}{ Pinky -thumb touch } \\
\hline $\mathrm{TP}=66$ & $\mathrm{FN}=4$ & 70 & $\mathrm{TP}=72$ & $\mathrm{FN}=17$ & 89 & $\mathrm{TP}=76$ & $\mathrm{FN}=4$ & 80 \\
\hline $\mathrm{FP}=18$ & $\mathrm{TN}=416$ & 434 & $\mathrm{FP}=12$ & $\mathrm{TN}=403$ & 415 & $\mathrm{FP}=8$ & $\mathrm{TN}=416$ & 424 \\
\hline 84 & 420 & 504 & 84 & 420 & 504 & 84 & 420 & 504 \\
\hline \multicolumn{3}{|c|}{$\mathrm{TPR}=0.942$} & \multicolumn{3}{|c|}{$\mathrm{TPR}=0.808$} & \multicolumn{3}{|c|}{ TPR $=0.95$} \\
\hline \multicolumn{3}{|c|}{$F P R=0.041$} & \multicolumn{3}{|c|}{$F P R=0.028$} & \multicolumn{3}{|c|}{$F P R=0.018$} \\
\hline \multicolumn{3}{|c|}{$P P V=0.785$} & \multicolumn{3}{|c|}{$P P V=0.857$} & \multicolumn{3}{|c|}{$P P V=0.904$} \\
\hline \multicolumn{3}{|c|}{$A C C=0.956$} & \multicolumn{3}{|c|}{$A C C=0.942$} & \multicolumn{3}{|c|}{$A C C=0.976$} \\
\hline
\end{tabular}

Table 6. Contingency matrixes.

The four outcomes can be formulated in a $2 \times 2$ contingency table. All contingency matrixes for each motion are shown in Table 6.

\section{3D modeling and manufacturing of prosthetic hand}

\subsection{D modeling of prosthetic hand via SolidWorks}

In order to develop a multifunctional prosthetic hand model, the structural characteristics of the human hand must first be determined. In other words, it is necessary to determine the number of joints, the number of links, the fingers and the length and width parameters of each finger. In order to obtain a prosthetic hand the same size as a human hand, the hand characteristics of an adult male were recorded as in Table 7 for the purposes of this study [42-44].

\begin{tabular}{lllllll}
\hline & First link & \multicolumn{2}{c}{ Second link } & \multicolumn{2}{c}{ Third link } \\
\cline { 2 - 6 } & Length $(\mathbf{m m})$ & Width $(\mathbf{m m})$ & Length $(\mathbf{m m})$ & Width $(\mathbf{m m})$ & Length $(\mathbf{m m})$ & Width $(\mathbf{m m})$ \\
\hline Thumb & 70 & 30 & 45 & 30 & 40 & 30 \\
Index & 55 & 30 & 40 & 25 & 30 & 25 \\
Middle & 55 & 30 & 50 & 25 & 40 & 25 \\
Ring & 55 & 30 & 40 & 25 & 30 & 25 \\
Pinky & 30 & 30 & 40 & 25 & 30 & 25 \\
Palm & 130 & 120 & & & & \\
\hline
\end{tabular}

Table 7. Part of the hand. 

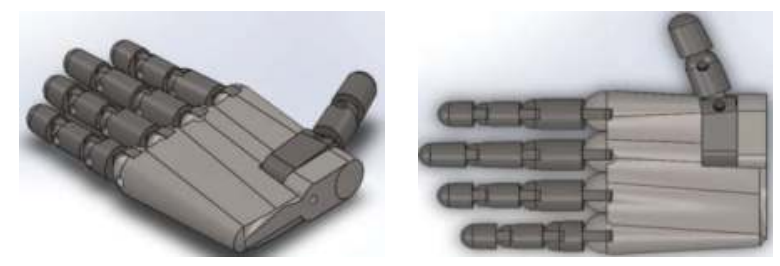

Figure 5. SolidWorks images of prosthetic hand.

Using the parameter values in Table 5, the prosthetic hand 3D model is designed with the help of the SolidWorks program as shown in Figure 5.

\subsection{Manufacturing of prosthetic hand via $3 \mathrm{D}$ printer}

The prototype of the prosthetic hand was produced with the help of the EDISON 3D printer manufactured by 3D Design Company. The necessary adjustments for the production (e.g., resolution, amount of fullness, amount of support) were made using the Simplify 3D program, which was offered by the same company as the software program. After a hand of 16 parts was produced, it was assembled as shown in Figure 6.
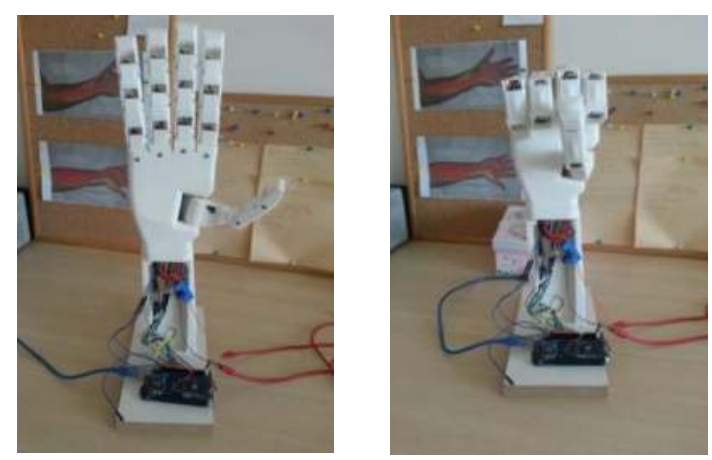

Figure 6. Prototype hand.

\section{Prosthetic hand simulator design}

\subsection{Mechanical design of prosthetic hand simulator via SimMechanics}

SimMechanics used in the realization of simulations of mechanical systems [45, 46]. By transferring the 3D CAD model of the prosthetic hand developed in the SolidWorks program to the Matlab SimMechanics program, a chain structure containing each joint and link of the prosthetic hand was obtained as shown in Figure 7. Five fingers connected to the palm, three rotary hinges forming each finger, and three connecting links are arranged in series to form the hand SimMechanics model. 


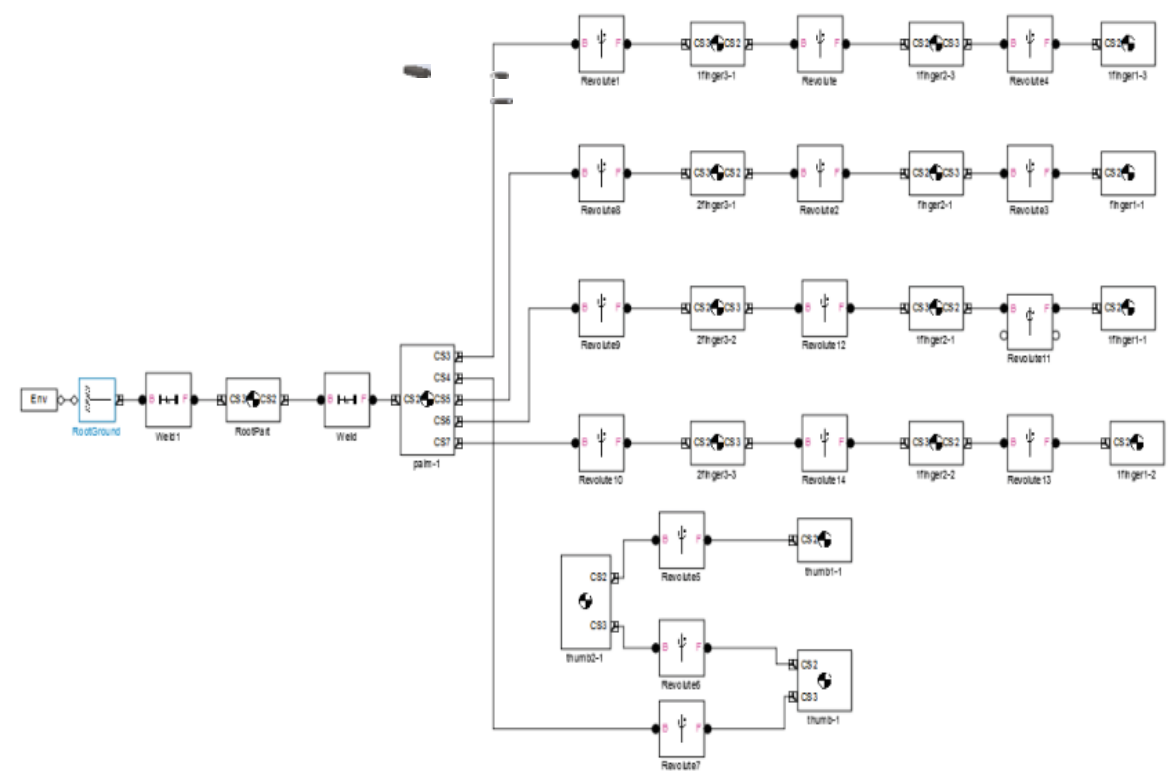

Figure 7. Prosthetic hand SimMechanics model.

As shown in Figure 7, when SolidWorks solid model is transferred to Matlab Program, a chain structure composed of revolute and link parts is obtained.

\subsection{Modeling of the DC motor}

In this study, it was decided to use a DC servo motor for movement of each joint in the prosthetic hand. The equivalent circuit of the DC servo motor is given in Figure 8 [47-49].

Modeling equations of DC motor were expressed in terms of the Laplace variable $s$ as Eqs. (11)-(13).

$$
\begin{gathered}
s(J s+B) \theta(s)=K_{t} I(s) \\
(L s+R) I(s)=V(s)-K_{e} s \theta(s)
\end{gathered}
$$

We arrive at the following open-loop transfer function by eliminating $I(s)$ between the two equations above, where the rotation is considered the output and the armature voltage is considered the input.

$$
\frac{\theta(s)}{V(s)}=\frac{K}{s\left((L s+R)(J s+b)+K^{2}\right)}
$$

Using the mathematical model of the DC servo motor, the Matlab/Simulink model is constructed as shown in Figure 9. 


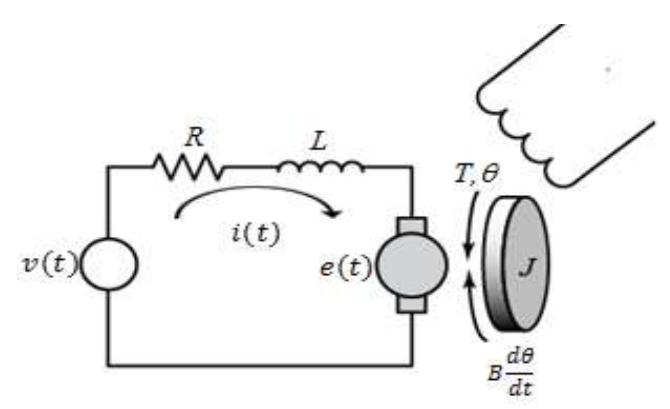

Figure 8. DC motor electrical and mechanical model.

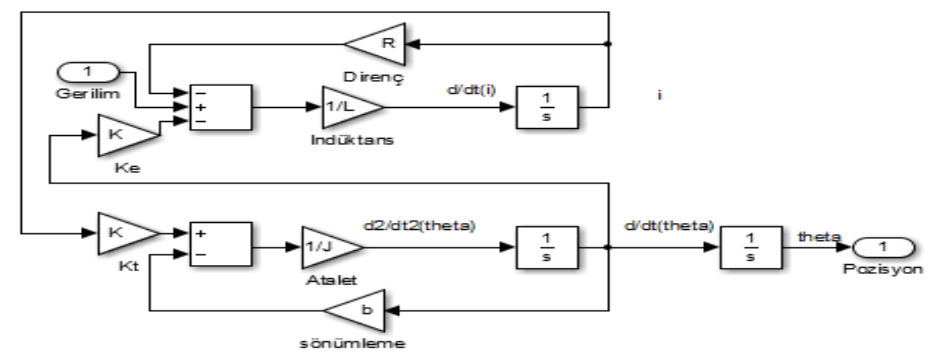

Figure 9. DC motor Matlab/Simulink model.

\section{Controller design}

Position of ultra-nano DC servomotors connected to joints is controlled using a PID controller. The controller's proportional gain coefficient $(\mathrm{Kp})$, integral gain coefficient $(\mathrm{Ki})$, and derivative gain $(\mathrm{Kd})$ values are determined by Genetic Algorithm [11,50-52] to ensure that the system quickly reaches a steady state without overshooting as shown in Table 8. The PID controller has an input-output relationship with input e (t) and output $u(t)$ [53-55].

$$
u(t)=K_{p} \cdot e(t)+K_{i} \cdot \int_{0}^{t} e(\tau) \cdot d \tau+K d \cdot \frac{d e(t)}{d t}
$$

\begin{tabular}{llll}
\hline & $\mathbf{K}_{\mathbf{p}}$ & $\mathbf{K}_{\mathbf{i}}$ & $\mathbf{K}_{\mathbf{d}}$ \\
\hline All DC motors connected the each finger joints & 0.42176 & 0.75724 & 0.0048566 \\
\hline
\end{tabular}

Table 8. PID parameters.

\section{Graphical and numerical results}

Electromyography is used to measure EMG signals, which are extracted from the forearm muscles and classified with the help of four surface electrodes. The type of motion that one wishes to perform is the perceived and designed three-dimensional prosthetic hand simulator 
and the five-fingered and 15-jointed hand. These movements were made in real time on the prototype. Each joint of the prosthetic hand is moved with one ultra-nano servomotor, and the position control of the motors is provided by the designed PID.

The prosthetic hand was made with hand closure, hand opening, thumb-index touch, hand opening, thumb-middle touch, hand opening, thumb-ring touch, hand opening, thumb-pinkie touch, and hand opening movements. The hand opening movement is performed after the hand closing movement and touch movement.

1. EMG signals were taken from four channels, four groups of muscles simultaneously, as shown in Figures 10-13, and preprocessed. First, the signal amplitude was scaled from 0 to $10 \mathrm{~V}$ and then filtered.
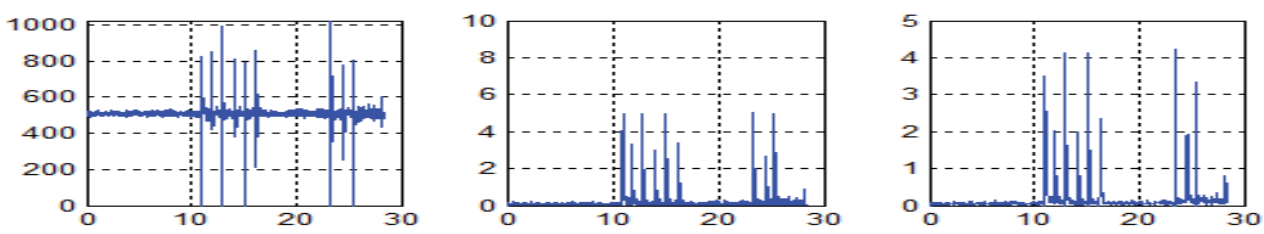

Figure 10. Preprocessing step graphics of EMG signal recorded Channel 1.
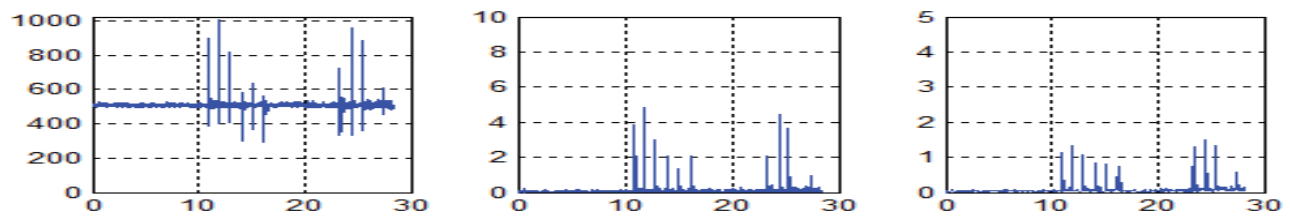

Figure 11. Preprocessing step graphics of EMG signal recorded Channel 2.
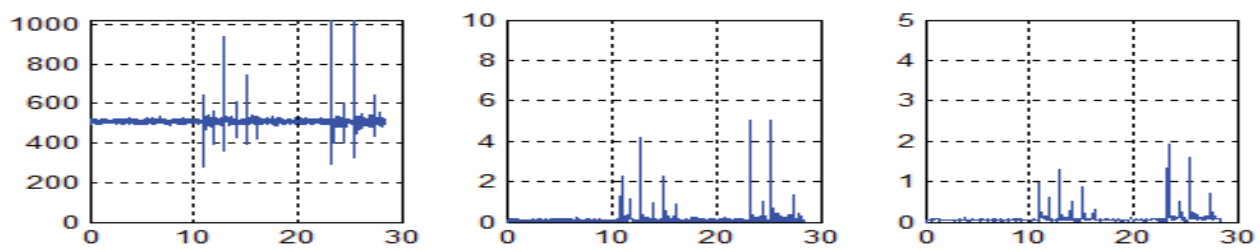

Figure 12. Preprocessing step graphics of EMG signal recorded Channel 3.
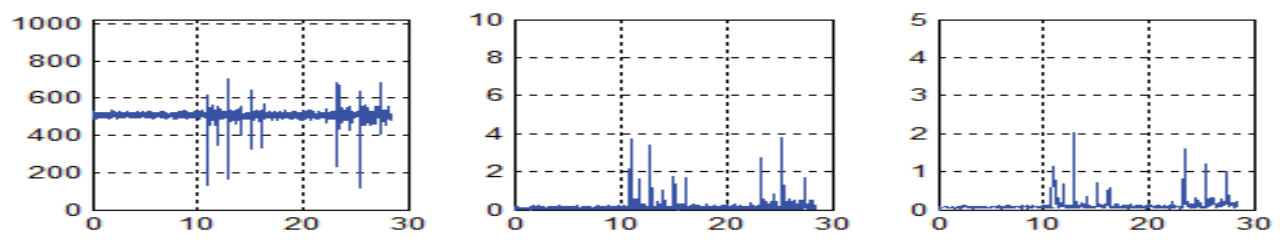

Figure 13. Preprocessing step graphics of EMG signal recorded Channel 4. 
2. As shown in Figures 14-17, the energy, maximum, effective, mean, and variance attribute values of the respective signals were calculated.

3. Motion pattern was determined by motion classification algorithm.

4. The specified type of motion information was input to the simulator and the prototype.

5. According to the recognized hand pattern, the reference joint angles in Table 9 were applied as the control input signal, and the closed loop position control of the DC servomotors was performed according to feedback information from sensors connected to the simulator joints.

Position control of the finger joints for six hand patterns was provided by the PID controllers as shown in Figures 18-23.

For all finger joints, PID performance is shown in Table 10.
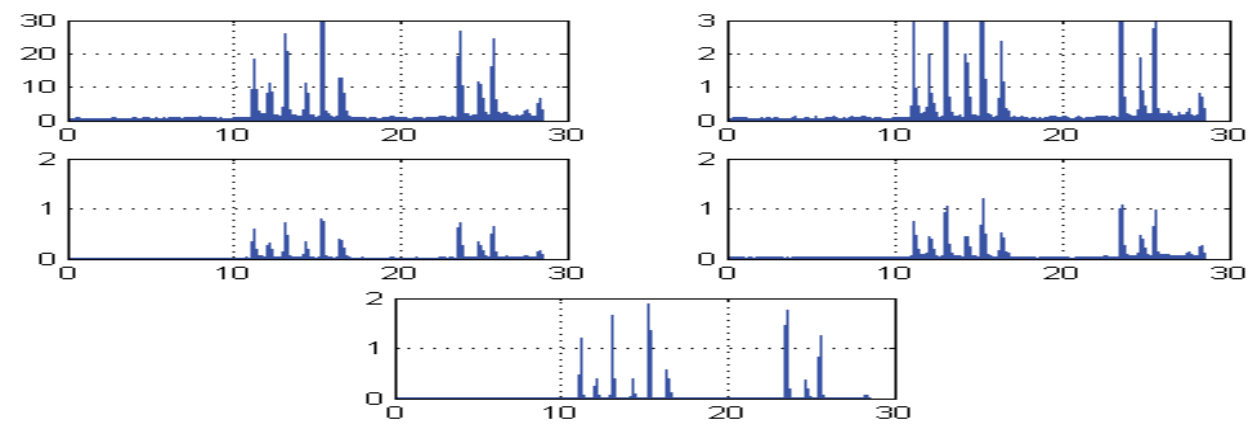

Figure 14. Features graphics of EMG signal recorded Channel 1.
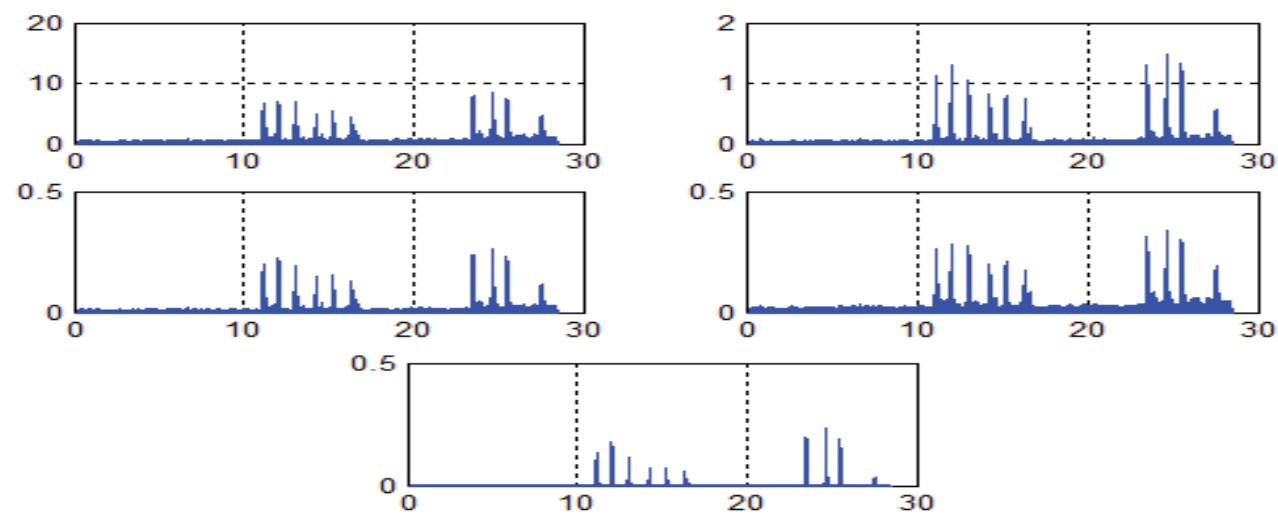

Figure 15. Features graphics of EMG signal recorded Channel 2. 

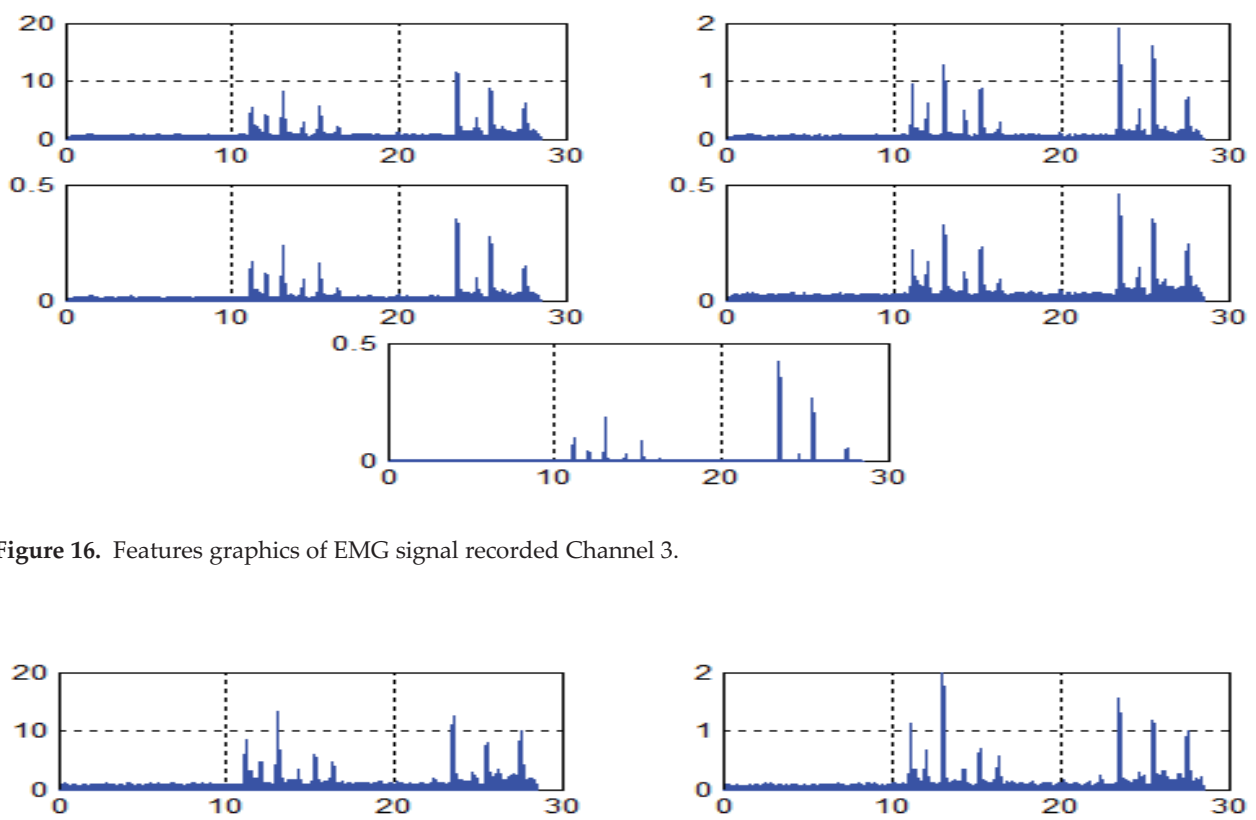

Figure 16. Features graphics of EMG signal recorded Channel 3.
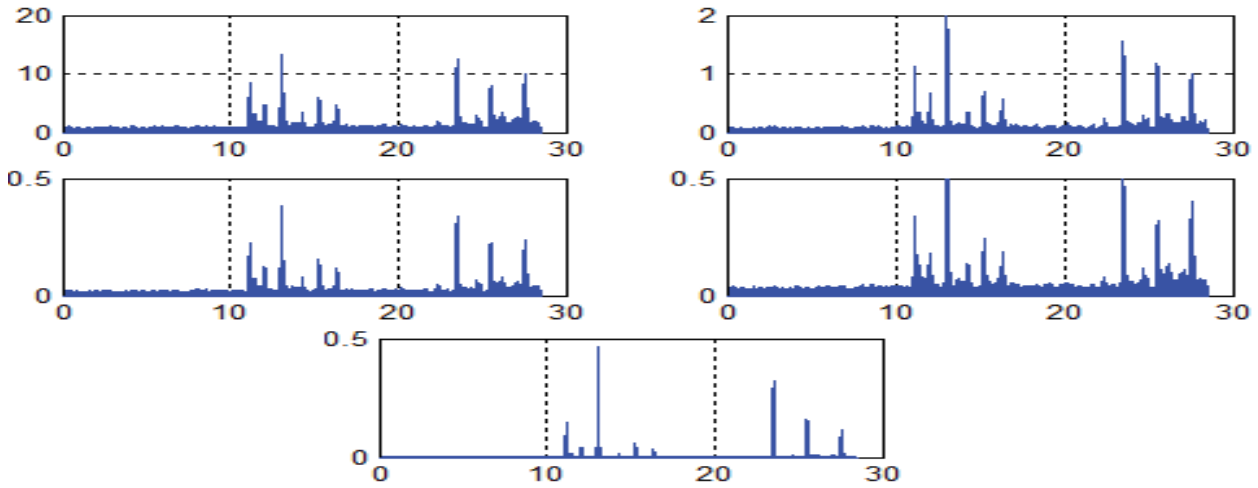

Figure 17. Features graphics of EMG signal recorded Channel 4.

\begin{tabular}{|c|c|c|c|c|c|c|c|c|c|c|c|c|c|c|c|c|}
\hline & & \multicolumn{3}{|c|}{ Index } & \multicolumn{3}{|c|}{ Middle } & \multicolumn{3}{|c|}{ Ring } & \multicolumn{3}{|c|}{ Pinkie } & \multicolumn{3}{|c|}{ Thumb } \\
\hline & & $\theta_{1}$ & $\theta_{2}$ & $\theta_{3}$ & $\theta_{1}$ & $\theta_{2}$ & $\theta_{3}$ & $\theta_{1}$ & $\theta_{2}$ & $\theta_{3}$ & $\theta_{1}$ & $\theta_{2}$ & $\theta_{3}$ & $\theta_{1}$ & $\theta_{2}$ & $\theta_{3}$ \\
\hline 1 & Motion 1 & 90 & 90 & 90 & 90 & 90 & 90 & 90 & 90 & 90 & 90 & 90 & 90 & 90 & 90 & 90 \\
\hline 2 & Motion 2 & 0 & 0 & 0 & 0 & 0 & 0 & 0 & 0 & 0 & 0 & 0 & 0 & 0 & 0 & 0 \\
\hline 3 & Motion 3 & 90 & 30 & 30 & 0 & 0 & 0 & 0 & 0 & 0 & 0 & 0 & 0 & 70 & 15 & 5 \\
\hline 4 & Motion 4 & 0 & 0 & 0 & 90 & 25 & 25 & 0 & 0 & 0 & 0 & 0 & 0 & 87 & 5 & 5 \\
\hline 5 & Motion 5 & 0 & 0 & 0 & 0 & 0 & 0 & 90 & 25 & 10 & 0 & 0 & 0 & 105 & 15 & 5 \\
\hline 6 & Motion 6 & 0 & 0 & 0 & 0 & 0 & 0 & 0 & 0 & 0 & 90 & 30 & 5 & 125 & 15 & 5 \\
\hline
\end{tabular}

Table 9. Reference value for each finger joints. 

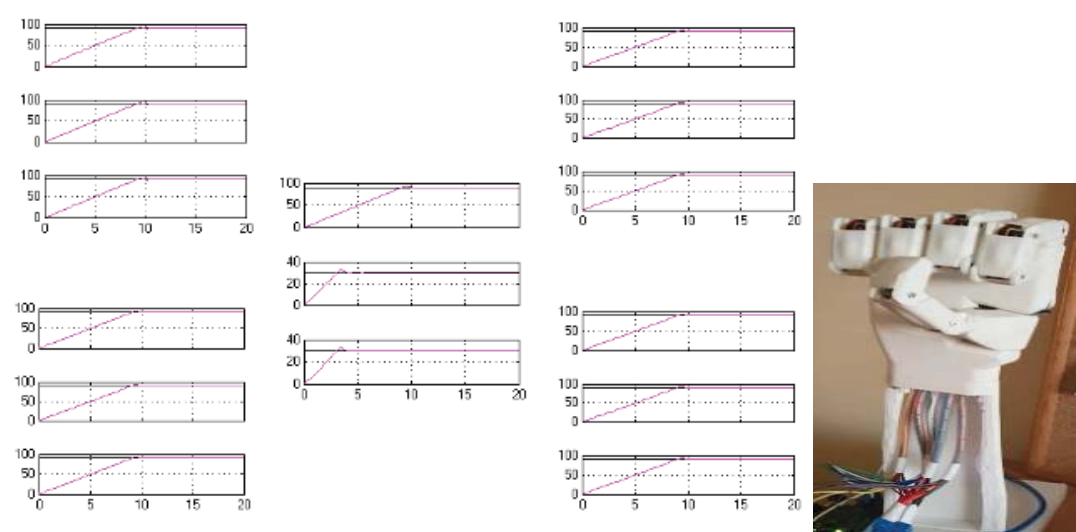

Figure 18. PID response graphics of five fingers for hand close and prosthetic hand photograph.
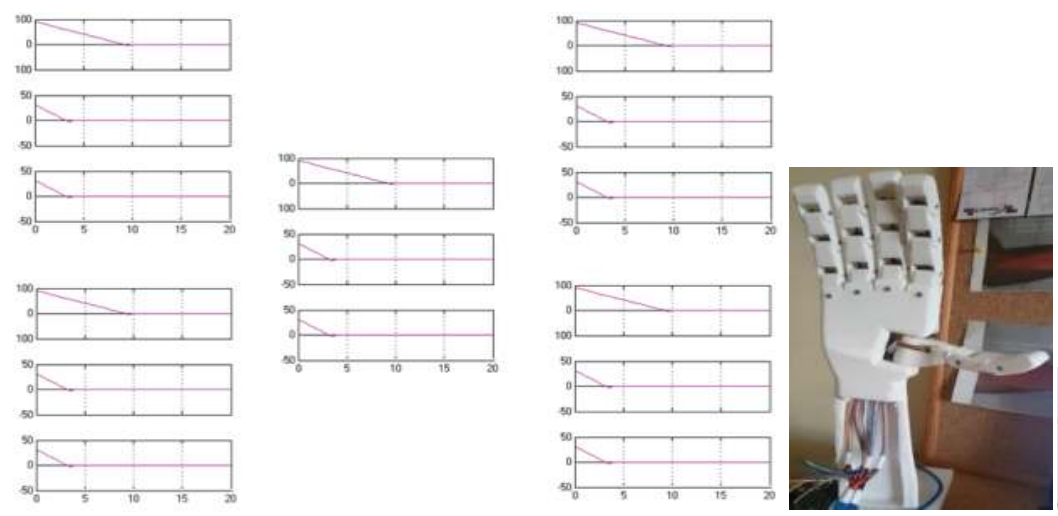

Figure 19. PID response graphics of five fingers for hand opening and prosthetic hand photograph.
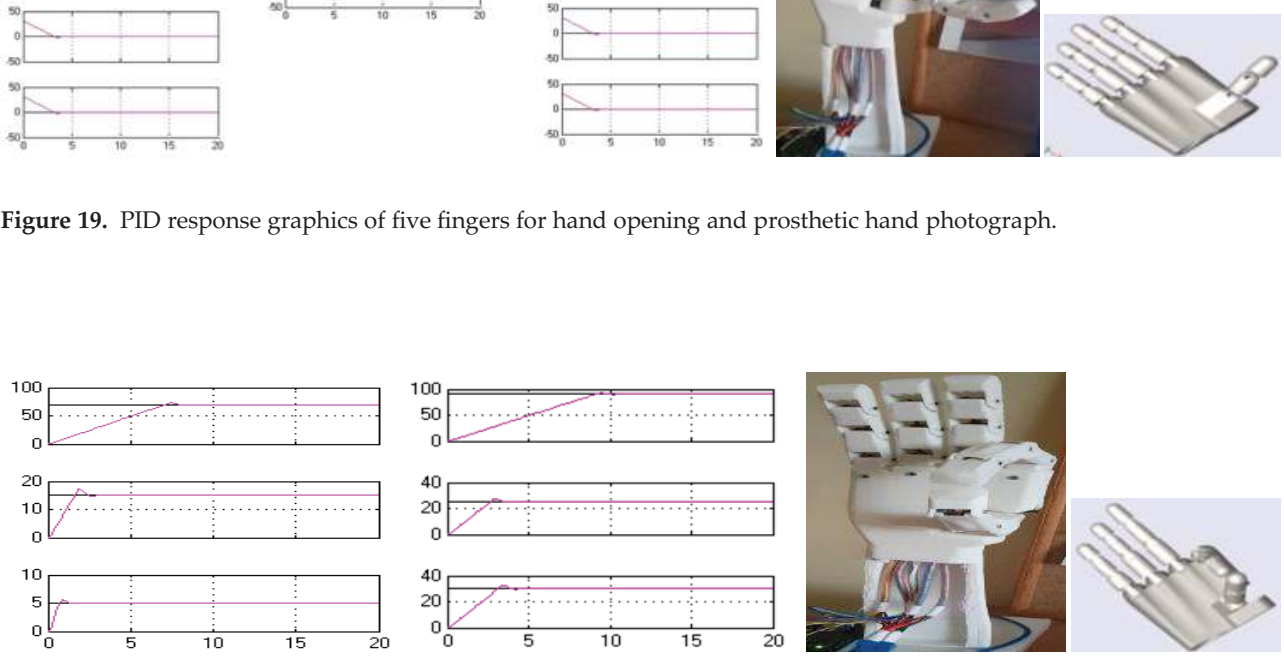

Figure 20. PID response graphics of five fingers for thumb-index touch and prosthetic hand photograph. 

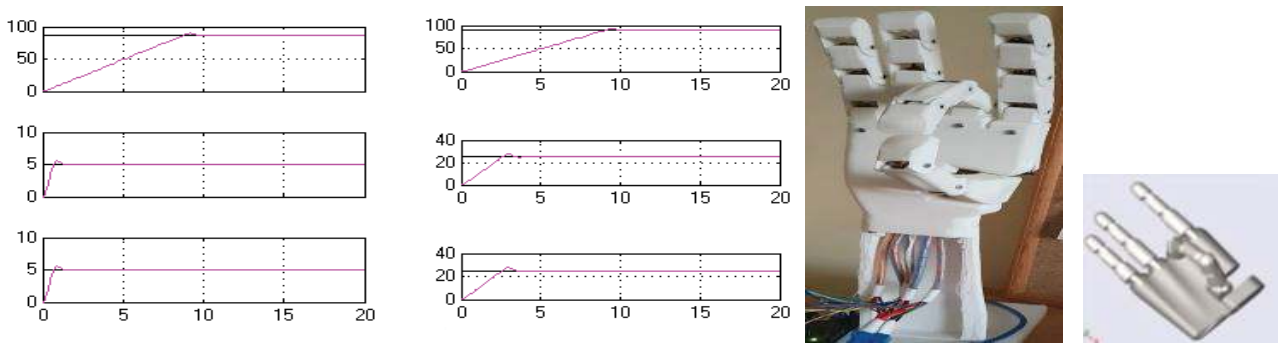

Figure 21. PID response graphics of five fingers for thumb-middle touch and prosthetic hand photograph.
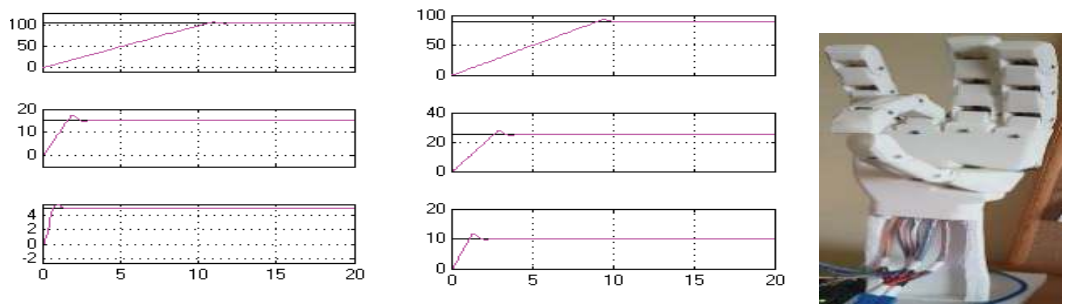

Figure 22. PID response graphics of five fingers for thumb-ring touch and prosthetic hand photograph.
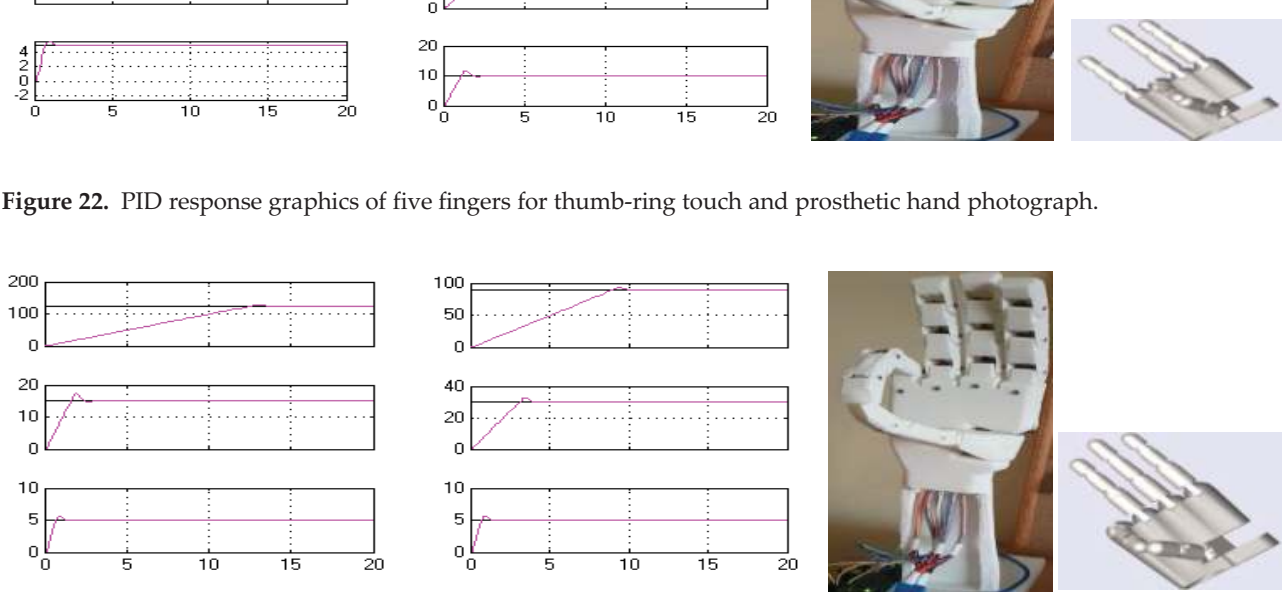

Figure 23. PID response graphics of five fingers for thumb-pinkie touch and prosthetic hand photograph.

\begin{tabular}{|c|c|c|c|c|c|c|c|c|}
\hline Finger & Joint no & & Motion 1 & Motion 2 & Motion 3 & Motion 4 & Motion 5 & Motion 6 \\
\hline \multirow[t]{9}{*}{ Thumb finger } & \multirow[t]{3}{*}{1} & Overshoot (deg.) & 2.835 & 0.2932 & 2.137 & 2.936 & 3.025 & 3.655 \\
\hline & & Steady state time (s) & 9.8084 & 13.413 & 8.8084 & 9.988 & 10.8084 & 12.8084 \\
\hline & & Steady state error (deg.) & 0.046 & 0.041 & 0.037 & 0.027 & 0.021 & 0.024 \\
\hline & \multirow[t]{3}{*}{2} & Overshoot (deg.) & 2.755 & 0.3265 & 0.652 & 0.252 & 0.652 & 0.652 \\
\hline & & Steady state time (s) & 4.415 & 2.883 & 1.952 & 0.752 & 1.952 & 1.952 \\
\hline & & Steady state error (deg.) & 0.0052 & $2.6 e-3$ & 0.0001 & 0.0001 & 0.0001 & 0.0001 \\
\hline & \multirow[t]{3}{*}{3} & Overshoot (deg.) & 2.754 & 0.2696 & 0.357 & 0.357 & 0.357 & 0.357 \\
\hline & & Steady state time (s) & 4.524 & 1.972 & 0.956 & 0.956 & 0.956 & 0.956 \\
\hline & & Steady state error (deg.) & 0.0053 & $1.5 \mathrm{e}-3$ & $1.5 \mathrm{e}-3$ & $1.5 \mathrm{e}-3$ & $1.5 \mathrm{e}-3$ & $1.5 e-3$ \\
\hline
\end{tabular}




\begin{tabular}{|c|c|c|c|c|c|c|c|c|}
\hline Finger & Joint no & & Motion 1 & Motion 2 & Motion 3 & Motion 4 & Motion 5 & Motion 6 \\
\hline \multirow[t]{9}{*}{ Index finger } & \multirow[t]{3}{*}{1} & Overshoot (deg.) & 2.835 & 0.3299 & 2.835 & 0 & 0 & 0 \\
\hline & & Steady state time (s) & 9.915 & 9.71 & 9.915 & 0 & 0 & 0 \\
\hline & & Steady state error (deg.) & 0.045 & $10 \mathrm{e}-4$ & 0.045 & 0 & 0 & 0 \\
\hline & \multirow[t]{3}{*}{2} & Overshoot (deg.) & 2.835 & 0.0368 & 2.349 & 0 & 0 & 0 \\
\hline & & Steady state time (s) & 9.915 & 4.555 & 7.0725 & 0 & 0 & 0 \\
\hline & & Steady state error (deg.) & 0.047 & 0.0183 & 0.0219 & 0 & 0 & 0 \\
\hline & \multirow[t]{3}{*}{3} & Overshoot (deg.) & 2.835 & 0.348 & 0.377 & 0 & 0 & 0 \\
\hline & & Steady state time (s) & 9.915 & 4.535 & 7.429 & 0 & 0 & 0 \\
\hline & & Steady state error (deg.) & 0.047 & 0.0202 & 0.0255 & 0 & 0 & 0 \\
\hline \multirow[t]{9}{*}{ Middle finger } & \multirow[t]{3}{*}{1} & Overshoot (deg.) & 2.8356 & 0.3244 & 0 & 2.8368 & 0 & 0 \\
\hline & & Steady state time (s) & 10.5022 & 10.279 & 0 & 10.52 & 0 & 0 \\
\hline & & Steady state error (deg.) & 0.0474 & $1 e-3$ & 0 & 0.0475 & 0 & 0 \\
\hline & \multirow[t]{3}{*}{2} & Overshoot (deg.) & 2.8356 & 0.3244 & 0 & 2.812 & 0 & 0 \\
\hline & & Steady state time (s) & 10.5022 & 10.279 & 0 & 3.437 & 0 & 0 \\
\hline & & Steady state error (deg.) & 0.0474 & $1 e-3$ & 0 & 0.0036 & 0 & 0 \\
\hline & \multirow[t]{3}{*}{3} & Overshoot (deg.) & 2.8356 & 0.3244 & 0 & 2.7812 & 0 & 0 \\
\hline & & Steady state time (s) & 10.5022 & 10.279 & 0 & 3.9265 & 0 & 0 \\
\hline & & Steady state error (deg.) & 0.0474 & $1 \mathrm{e}-3$ & 0 & 0.0036 & 0 & 0 \\
\hline \multirow[t]{9}{*}{ Ring finger } & \multirow[t]{3}{*}{1} & Overshoot (deg.) & 2.8356 & 0.3244 & 0 & 0 & 2.8368 & 0 \\
\hline & & Steady state time (s) & 9.922 & 9.907 & 0 & 0 & 9.914 & 0 \\
\hline & & Steady state error (deg.) & 0.047 & $1 e-3$ & 0 & 0 & 0.047 & 0 \\
\hline & \multirow[t]{3}{*}{2} & Overshoot (deg.) & 2.8356 & 0.3244 & 0 & 0 & 2.781 & 0 \\
\hline & & Steady state time (s) & 9.915 & 9.9075 & 0 & 0 & 3.412 & 0 \\
\hline & & Steady state error (deg.) & 0.047 & $1 e-3$ & 0 & 0 & 0.0035 & 0 \\
\hline & \multirow[t]{3}{*}{3} & Overshoot (deg.) & 2.8357 & 0.3244 & 0 & 0 & 2.545 & 0 \\
\hline & & Steady state time (s) & 9.9055 & 9.906 & 0 & 0 & 1.884 & 0 \\
\hline & & Steady state error (deg.) & 0.047 & $1 e-3$ & 0 & 0 & 0.0005 & 0 \\
\hline \multirow[t]{9}{*}{ Pinkie finger } & \multirow[t]{3}{*}{1} & Overshoot (deg.) & 2.8356 & 0.3244 & 0 & 0 & 0 & 2.8368 \\
\hline & & Steady state time (s) & 9.9094 & 9.9122 & 0 & 0 & 0 & 9.29 \\
\hline & & Steady state error (deg.) & 0.0475 & $1 \mathrm{e}-3$ & 0 & 0 & 0 & 0.0475 \\
\hline & \multirow[t]{3}{*}{2} & Overshoot (deg.) & 2.8357 & 0.3244 & 0 & 0 & 0 & 2.7883 \\
\hline & & Steady state time (s) & 9.9094 & 9.9122 & 0 & 0 & 0 & 4.8174 \\
\hline & & Steady state error (deg.) & 0.0475 & $1 e-3$ & 0 & 0 & 0 & 0.0052 \\
\hline & \multirow[t]{3}{*}{3} & Overshoot (deg.) & 2.8357 & 0.3244 & 0 & 0 & 0 & 2.636 \\
\hline & & Steady state time (s) & 9.9094 & 9.9122 & 0 & 0 & 0 & 1.3391 \\
\hline & & Steady state error (deg.) & 0.0475 & $1 \mathrm{e}-3$ & 0 & 0 & 0 & 0 \\
\hline
\end{tabular}

Table 10. PID performance value for each joint. 


\section{Conclusion}

The main factor in increasing the functionality of the prosthetic hand to the extent of imitating biological hand functions is the movement of the fingers. The greater the number of movements the fingers can do independently of each other, the greater the ability of the prosthetic hand to move and the more successfully it can mimic the biological hand. Within the scope of this thesis, the function of the prosthetic hand is improved by six different finger movements. Bioelectrical signals of two separate users were recorded from the forearm muscles (the flexor pollicis longus, flexor carpi radialis, brachioradialis, extensor carpi radialis, extensor digiti minimi, and extensor carpi ulnaris) with the help of four surface electrode groups. Thus, a broad bioelectrical signal database was created. The recorded bioelectrical signals were subjected to a series of preprocessing and feature extraction processes to calculate the maximum, effective, mean, variance, and energy values of the EMG signals. An FL classification algorithm was developed to create an effective cognitive interaction network, and 90\% classification success was obtained from these algorithms. The identified bioelectrical signals were applied to the designed three-dimensional prosthesis handheld simulator. The five-fingered and 15 -jointed prosthetic hand prototypes produced with a $3 \mathrm{D}$ printer, and the positional control of the prosthetic finger joints was performed with the designed controllers. Each finger of the prosthetic hand was moved by an ultra-nano DC motor, and the position controls of the motors were provided by the designed PID. Thus, a cognitive interface and communication network were established between the person and the prosthetic hand with great success.

\section{Acknowledgements}

The subject of this chapter, which is Beyda TAŞAR's doctoral thesis, was supported by TÜBİTAK under the Domestic Doctoral Scholarship Program for Priority Areas in 2211 C. In addition, the study was supported by Furat University Scientific Research Projects Management Unit within the scope of PhD Thesis Project number MF-14.25.

\section{Author details}

Beyda $\operatorname{Taşar}^{1 *}$ and Arif Gülten ${ }^{2}$

*Address all correspondence to: btasar@firat.edu.tr

1 Firat University, Engineering Faculty, Department of Mechatronics, Elazig, Turkey

2 Firat University, Engineering Faculty, Department of Electrical and Electronics, Elazig, Turkey

\section{References}

[1] Boostani R, Moradi MH. Evaluation of the forearm EMG signal features for the control of a prosthetic hand. Institute of Physics Publishing Physiological Measurement. Physiological Measurement. 2003;24:309-319. PII: S0967-3334(03)38946-4 
[2] Nishikawa D, Yu W, Yokoi H, Kakazu Y. On-line supervising mechanism for learning data in surface electromyogram motion classifiers. Journal and Communication in Japan. 2002;33:14, pp. 2634-2643

[3] Arieta AH, Katoh R, Yokoi H, Wenwei Y. Development of multi-DOF electromyography prosthetic system using the adaptive joint mechanism. Applied Bionics and Biomechanics. 2006;3:101-112

[4] Zhao J, XIie Z, Jing L, Cai H, Hong L, Hirzınger G. EMG control for a five-fingered prosthetic hand based on wavelet transform and autoregressive model. International Conference on Mechatronics and Automation, China; 2006.

[5] Carrozza MC, Cappiello G, Stellin G, Zaccone F, Vecchi F, Micera S, Dario P. On the development of a novel adaptive prosthetic hand with compliant joints: Experimental platform and EMG control. In: Proceedings of the IEEE/RSJ International Conference on Intelligent Robots and Systems; April 2005. pp. 3951-3956

[6] Hocaoğlu E. Data acquisition and feature extraction for classification of prehensile SEMG signal for control of a multifunctional prosthetic hand [Master thesis]. Istanbul: Sabanci University, Mechanical Engineering; 2010.

[7] Carozza M, Cappiello G, Stellin G, Zaccone F, Vecchi F, Micera S, Dario P., On the development of a novel adaptive prosthetic hand with compliant joints: Experimental platform and EMG control, Intelligent Robots and Systems, 2005.(IROS 2005). pp. 12711276, 2-6 August 2005, 10.1109/IROS.2005.1545585

[8] Taylor Cl, Schwarz RJ. The anatomy and mechanics of the human hand. Artificial Limbs 2.2. 1955:22.

[9] Rakibul H, Vepar SH, Hujijbert H. Modeling and control of the Barrett hand for grasping. In: 15th International Conference on Computer Modelling and Simulation; 10-12 April 2013; Cambridge University.

[10] Hasan R, Rahideh A, Shaheed H. Modeling and interactional of the multifingered hand. In: Proceedings of the 19th International Conference on Automation \& Computing. Brunel University, Uxbridge, UK; 2013.

[11] Michalewicz, Z. Genetic Algorithms + Data Structures = Evolution Programs. Berlin Heidelberg, New York: Springer-Verlag; 1999.

[12] Widhiada W, Douglas SS, Jenkinson ID, Gomm JB. Design and control of three fingers motion for dexterous assembly of compliant elements. International Journal of Engineering, Science and Technology. 2011;3(6):18-34

[13] Al-Assaf Y, Al-Nashash H. Surface myoelectric classification for prostheses control. Journal of Medical Engineering \& Technology. 2005;29(5):203-207

[14] Parker P, Englehart K, Hudgins B. Myoelectric signal processing for control of powered limb prostheses. Journal of Electromyography and Kinesiology. 2006;16:541-548 
[15] Englehart K, Hudgins B. A robust, real-time control scheme for multifunction myoelectric control. IEEE Transactions on Biomedical Engineering. 2003;50(7):848-854

[16] Hudgins B, Parker P, Scott RN. A new strategy for multifunction myoelectric control. IEEE Transactions on Biomedical Engineering. 1993;40(1):82-94

[17] Asghari Oskoei M, Hu H. Myoelectric control systems - a survey. Biomedical Signal Processing and Control. 2007;4(4):275-294

[18] Lamounier E, Soares A, Andrade A, Carrijo R. A virtual prosthesis control based on neural networks for EMG pattern classification. In: Proceedings of the Artificial Intelligence and Soft Computing; Canada; 2002

[19] Chu J, Moon I, Kim S, Mun M. Control of multifunction myoelectric hand using a real time EMG pattern recognition. In: Proceedings of the IEEE/RSJ International Conference on Intelligent Robots and Systems; China; 2005. pp. 3957-3962

[20] Mohammadreza AOI, Huosheng H. Review: Myoelectric control systems: A survey. Biomedical Signal Processing and Control. 2007;2:275-294

[21] Zhao Z, Chen X, Zhang X, Yang J, Tu Y, Lantz V, Wang K. Study on Online Gesture SEMG Recognition, Advanced Intelligent Computing Theories and Applications. With Aspects of Theoretical and Methodological Issues. 2007:1257-1265

[22] Mahdi K, Mehran J. A novel approach to recognize hand movements via sEMG patterns. Engineering in Medicine and Biology Society. In: 29th Annual International Conference of the IEEE; 2007.

[23] Zhizeng L, Xiaoliang R, Yutao Z. Multi-pattern recognition of the forearm movement based on SEMG, Information Acquisition. In: Proceedings of the International Conference on IEEE; 21-25 June 2004. DOI: 10.1109/ICIA.2004.1373391

[24] Roberto M. Electromyography Physiology, Engineering and Noninvasive Applications. IEEE Press, John Wiley \& Sons Inc.; 2004, New Jersey, Canada

[25] De Luca CJ. Electromyography. Encyclopedia of Medical Devices and Instrumentation, John Wiley Publisher; 2006. pp. 98-109, New York City, United States, DOI: 10.1002/ 0471732877.emd097

[26] Hargrove L, Englehart K, Hudgins B. The effect of electrode displacements on pattern recognition based myoelectric control. In: IEEE Annual International Conference on Engineering in Medicine and Biology Society; 2006. pp. 2203-2206

[27] De Luca CJ. The use of surface electromyography in biomechanics. Journal of Applied Biomechanics. 1997;1(2):135-163

[28] SENIAM EMG protocol. Available from: http://www.seniam.org/(Download date: 21.03.2014)

[29] Daud WMBW, Yahya AB, Horng CS, Sulaima MF, Sudirman R. Features extraction of electromyography signals in time domain on biceps brachii muscle. International Journal of Modeling and Optimization. 2013;3(6) 
[30] Yazıcı İ. EMG İşaretlerinin İşlenmesi Ve Sınıflandırılması [Master thesis]. Sakarya: Sakarya University, Institute of Science and Technology; 2008

[31] Taşan D. Protez Denetimi İçin Elektromiyografi (EMG)de Örüntü Tanıma, Yüksek Lisans Tezi, Ege Universty, Institute of Science and Technology, İzmir, 2008

[32] Akgün G, Demetgül M, Kaplanoğlu E. EMG Sinyallerinin Öznitelik Çıkarımı ve Geri Yayılımlı Yapay Sinir Ağı, Algoritması İle Sınıflandırılması, Otomatik Kontrol Ulusal Toplant1s1, TOK2013, Malatya 26-28 Eylül 2013.

[33] Chen WT, Wang Z, Ren X. Characterization of surface EMG signals using improved approximate entropy. Zhejiang University Science B. 2006;7(10): 844-848

[34] Chu J, Moon I, Mun M. A real-time EMG pattern recognition system based on linear-nonlinear feature projection for a multifunction myoelectric hand. IEEE Transactions on Biomedical Engineering. 2006;53:2232-2238

[35] Chan F, Yong-Sheng Y, Lam F, Yuan-Ting Z, Parker P. Fuzzy EMG classification for prosthesis control. IEEE Transactions on Rehabilitation Engineering. 2000;8(3)

[36] Yaraş B, Hüseynov R, Namazov M, ÇeliKKale IE, Şeker M. Fuzzy control and sliding mode fuzzy control of DC motor. Journal Of Engineering And Natural Sciences Mühendislik Ve Fen Bilimleri Dergisi, Sigma. 2014;32:97-108

[37] Ajiboye A, Weir R. A heuristic fuzzy logic approach to EMG pattern recognition for multifunction prosthesis control. IEEE Transactions on Biomedical Engineering. 2005;52 (11): 280-291

[38] Karlik B, M.O., T., \& M., A. A fuzzy clustering neural network architecture for multifunction upperlimb prosthesis. IEEE Transactions on Biomedical Engineering. 2003;50:1255-1261

[39] Vuskovic M, Du SJ. Classification of prehensile EMG patterns with simplified fuzzy ARTMAP networks. In: Proceedings of the International Joint Conference on Neural Networks; 2002, 3, pp. 2539-2544

[40] Khzeri M, Jahed M, Sadati N. Neuro-fuzzy surface EMG pattern recognition for multifunctional hand prosthesis control, Industrial Electronics. In: IEEE International Symposium; 2007

[41] Zheng L, He X. Classification techniques in pattern recognition. In: WSCG, Conference Proceedings; 2005, ISBN 80-903100-8-7

[42] Taşar B, Yakut O, Gülten A. Object detection and grip force control via force sensor for EMG based prosthesis hand, E205. In: International Conference on Electrical and Electronics Engineering (ICEEE); 27-28 Nisan 2015; Ankara

[43] Taşar B, Gülten A, Yakut O. Modeling controlling and simulation of 15 DOF multifunctional prosthesis hand using SimMechanics, C201. In: International Conference on Automatic Control (ICOAC); 27-28 Nisan 2015; Ankara 
[44] Taşar B, Gülten A, Yakut O. Kinematic analysis of the human hand for desing prosthetic hand. In: 3rd International Congress on Natural and Engineering Sciences; September 913, 2015; Sarajevo, Bosnia and Herzegovina: International University of Sarajevo

[45] Dung LT, Kang HJ, Ro YS. Robot manipulator modeling in Matlab SimMechanics with PD control and online Gravity compensation. IFOST Proceedings. 2010

[46] Fedák V, Durovsky F, Üveges R. Analysis of robotic system motion in SimMechanics and MATLAB GUI Environment. MATLAB Applications for the Practical Engineer. Chapter 20. 2014

[47] Bencsik AL. Appropriate mathematical model of DC servo motors applied in SCARA robots. Acta Polytechnica Hungarica. 2004;1(2):2004-2099

[48] Matlan Tuturial, DC Motor Position Simulink Model. Available from: Dowload:http:// ctms.engin.umich.edu/CTMS/index.php?example=MotorPosition\&section=SimulinkModeling

[49] Meier R. Modeling DC servo motors control systems, Tech Note, Milwaukee School of Engineering 1025 North Broadway, Milwaukee. https://faculty-web.msoe.edu/meier/ ee3720/technotes/dcservo.pdf

[50] Haupt Randly L, Haupt Sue E. Practical Genetic Algorithms. USA: A Wiley-Interscience Publication; 1998

[51] Davis L. Handbook of Genetic Algorithms. New York, NY: Van Nostrand; 1991

[52] Wook C, Ramakr Shna RS. Elitism-based compact genetic algorithms. IEEE Transactions on Evolutionary Computation. 2003;7(4):367-385

[53] Astrom KJ, Hagglund T. PID Controllers: Theory, Design and Tuning. Instrument Society of America; 1995. p. 343, Research Triangle Park, North Carolina, 1995

[54] Taguchi H, Araki M. Two-degree-of-freedom PID controllers. Proceedings of the IFAC Workshop on Digital Control: Past, Present and Future of PID Control; Elsevier; pp. 9196. 5-7 April 2000, Terrassa, Spain

[55] Bennett S. A History of Control Engineering, Peter Peregrinus; 1993. pp. 28-69, London, United Kingdom 
